\title{
Surveillance de la rougeole au Canada, 2015
}

\author{
Sherrard $\mathrm{L}^{1 *}$, Hiebert $\mathrm{J}^{2}$, Cunliffe $\mathrm{J}^{1}$, Mendoza $\mathrm{L}^{2}$, Cutler $\mathrm{J}^{1}$
}

\section{Résumé}

Contexte : La rougeole ne sévit plus au Canada depuis 1998. Chaque année, l'Agence de la santé publique du Canada (ASPC) présente des données épidémiologiques à l'Organisation panaméricaine de la Santé (OPS) en vue de vérifier que l'élimination de la rougeole persiste au Canada.

Objectif : Faire état de l'activité rougeoleuse au Canada en 2015, à l'aide de données probantes à jour étayant le statut d'élimination durable de la rougeole au Canada.

Méthodologie : Les données de surveillance de la rougeole ont été saisies par le Système canadien de surveillance de la rougeole et de la rubéole (SCSRR) et le projet pilote sur la surveillance de la rougeole et de la rubéole et évaluées pour la répartition en fonction des caractéristiques démographiques et des facteurs de risque. On a rédigé un résumé des caractéristiques des éclosions, et mené et décrit des analyses génotypiques et phylogéniques. Les données de surveillance pour l'année 2015 ont été évaluées par rapport aux critères essentiels d'évaluation de l'élimination de la rougeole de l'Organisation panaméricaine de la Santé.

Résultats : En 2015, l'incidence de la rougeole au Canada était de 5,5 cas pour 1000000 d'habitants, avec 196 cas dans quatre provinces. La majorité des cas $(87,2 \%, n=171)$ n'étaient pas immunisés, et les taux d'incidence propres à l'âge ainsi que les nombres de cas étaient plus élevés chez les personnes âgées de 10 à 14 ans (29,5 cas pour 1000000 d'habitants, $n=55)$. Cette situation était attribuable en grande partie à une éclosion notable dans une communauté religieuse ne pratiquant pas la vaccination. Dans l'ensemble, $10,7 \%(n=21)$ des cas ont été hospitalisés. Les renseignements sur le génotype étaient accessibles pour $100 \%$ des cas de rougeole (4 éclosions sur 4, et 6 cas sporadiques sur 6 ). Le Canada a satisfait, pleinement ou partiellement, à la plupart des critères de l'Organisation panaméricaine de la Santé pour la vérification de l'élimination de la rougeole.

Conclusion : Bien que les cas importés et les zones à faible couverture vaccinale continuent de menacer le statut d'élimination du Canada, les données de surveillance pour 2015 fournissent des preuves solides que l'élimination de la rougeole a été maintenue.

\author{
Affiliations \\ ${ }^{1}$ Centre de l'immunisation \\ et des maladies respiratoires \\ infectieuses, Agence de la santé \\ publique du Canada, Ottawa \\ (Ontario) \\ 2 Laboratoire national de \\ microbiologie, Agence de la santé \\ publique du Canada, Winnipeg \\ (Manitoba)
}

*Correspondance : lindsey. sherrard@phac-aspc.gc.ca

Citation proposée : Sherrard L, Hiebert J, Cunliffe J, Mendoza L, Cutler J. Surveillance de la rougeole au Canada, 2015. Relevé des maladies transmissibles au Canada 2016;42:155-62. https://doi.org/10.14745/ccdr.v42i07a01f

\section{Introduction}

La rougeole est I'une des maladies connues les plus contagieuses. Avant que les vaccins contre la rougeole deviennent largement accessibles, la maladie était une importante cause de décès et d'invalidité dans le monde entier, causant 2,6 millions de décès chaque année, selon les estimations (1).

Au Canada, la rougeole a été une maladie à déclaration obligatoire depuis 1924, sauf de 1959 à 1968. Une surveillance accrue fondée sur les cas de rougeole est coordonnée par le Centre de l'immunisation et des maladies respiratoires infectieuses et le Laboratoire national de microbiologie (LNM) de l'Agence de la santé publique du Canada, par le biais du Système canadien de surveillance de la rougeole et de la rubéole (SCSRR) et du projet pilote sur la surveillance de la rougeole et de la rubéole. La surveillance accrue de la rougeole est nécessaire afin de fournir des preuves suffisantes de l'élimination de la rougeole.

L'élimination de la rougeole est définie comme l'absence de transmission de la rougeole endémique dans une zone géographique définie pendant 12 mois ou plus, au moyen d'un système de surveillance bien rodé (2). L'élimination de la rougeole au Canada a été décrite comme un important objectif de santé publique atteignable depuis au moins 1980 (3). Au cours de la Conférence de concertation de 1992 sur la rougeole, le Canada s'était donné comme objectif d'avoir éliminé la rougeole en 2005 (4). Cet objectif a été révisé à l'occasion de la Conférence panaméricaine sur la santé XXIV de 1994, alors que le Canada et d'autres États membres se sont entendus pour 
éliminer la rougeole dans les Amériques au plus tard en 2000 (5). À la suite de la mise en œuvre d'un programme de vaccination systématique à deux doses contre la rougeole, le dernier cas endémique au Canada a été signalé en 1997, et le statut d'élimination de la rougeole a été obtenu un an plus tard (6).

Malgré ce succès, le statut d'élimination du Canada continue d'être menacé par l'importation de la rougeole d'autres pays où la maladie demeure endémique. Afin de vérifier son statut d'élimination de la rougeole sur une base continue, le Canada soumet des données de surveillance à l'Organisation panaméricaine de la Santé. L'objectif du présent rapport est de fournir un résumé épidémiologique de l'activité rougeoleuse observée au Canada pour l'année épidémiologique 2015.

\section{Méthodologie}

Données de surveillance : Les provinces et les territoires ont signalé hebdomadairement à I'ASPC, par le biais du SCSRR ou du projet pilote de surveillance de la rougeole et de la rubéole, les cas de rougeole correspondants à la définition nationale d'un cas (7) [ $n=10$ dans les provinces et $n=3$ dans les territoires], y compris la déclaration de "zéro cas ». Les données non nominatives et sans identification sur les cas ont été extraites et soumises à l'Organisation panaméricaine de la Santé. Les cas confirmés de rougeole avec une éruption cutanée apparue au cours de l'année épidémiologique 2015 (du 4 janvier 2015 au 2 janvier 2016) ont été inclus dans le présent rapport.

Génotypage : Tous les génotypages du virus de la rougeole ont été effectués au Laboratoire national de microbiologie de l'Agence. On a effectué - par transcription inverse-amplification en chaîne de la polymérase (TI-ACP) le génotypage normalisé de l'Organisation mondiale de la Santé (OMS), soit le séquençage des 450 nucléotides du gène (région N-450) de la nucléoprotéine et du gène entier de l'hémagglutinine [gène $\mathrm{H}$ ] (8), de tous les cas de rougeole confirmés. Les échantillons cliniques (respiratoires et/ou urinaires) ont été transmis au Laboratoire national de microbiologie par les laboratoires provinciaux et ont été confirmés par TI-ACP dans les laboratoires provinciaux ou au Laboratoire national de microbiologie. Les séquences de la région $\mathrm{N}-450$ et du gène $\mathrm{H}$ ont été comparées aux séquences de référence des génotypes de l'OMS (9), et des arbres phylogénétiques de parcimonie maximale ont été générés au moyen du logiciel MEGA6 (10). Les génotypes ont été attribués en fonction de l'homologie maximale des séquences $\mathrm{N}-450$ par rapport aux génotypes de référence de l'Organisation mondiale de la Santé (9). Les séquences ont également été versées dans la base de données Measles Nucleotide Surveillance (MeaNS) de l'OMS (http://www. who-measles.org), comparées à des "souches nommées " ainsi qu'à des séquences déposées par d'autres membres du Réseau mondial de laboratoires pour la rougeole $(9,11)$.

Gestion et validation des données : Les données de surveillance de la rougeole ont été gérées à l'aide du logiciel Microsoft Access 2010. Un processus de validation des données a été réalisé en mars 2016 avec les quatre provinces qui avaient signalé des cas de rougeole en 2015. Ce processus comprenait une interrogation à propos des champs vides, la détermination des saisies de champ illogiques et la confirmation des valeurs auprès les territoires de compétence ayant effectué les déclarations.

Analyse : Le logiciel Enterprise Guide de SAS 5.1 (12) a été utilisé pour effectuer les analyses épidémiologiques descriptives, ainsi que pour le calcul des variables catégoriques (nombres, proportions) et des variables continues (médianes, intervalles). Les taux d'incidence ont été calculés en fonction des estimations de la population de Statistique Canada en date du 1 er juillet 2015. On a évalué la répartition des cas de rougeole selon les caractéristiques démographiques ( $p$. ex. âge, sexe, lieu), les signes de risque (p. ex. état vaccinal, hospitalisation, source d'exposition) et le génotype. Les caractéristiques des éclosions ont été résumées et les données de surveillance ont été comparées aux critères essentiels pour le maintien du statut d'élimination de la rougeole décrits par l'Organisation panaméricaine de la Santé (13).

L'état vaccinal était défini conformément au calendrier d'immunisation systématique financé par l'État (14). Les cas qui n'étaient pas admissibles au programme d'immunisation systématique en raison de leur âge (c.-à-d. âgés de moins d'un an ou qui sont nés avant 1970) ont été classés dans la catégorie " à jour ", sans égard à leur état déclaré. Les personnes nées après 1970 et âgées de sept ans ou plus ont été définies comme étant à jour si elles avaient reçu deux doses. En ce qui concerne les enfants âgés de 1 à 6 ans, l'administration d'une ou de deux doses, selon le calendrier recommandé dans la province ou le territoire de déclaration, les qualifiait parmi les sujets à jour.

Cette activité systématique de surveillance de la santé publique n'avait pas à être approuvée par un comité d'examen éthique.

\section{Résultats}

\section{Aperçu}

En 2015, l'incidence de la rougeole au Canada était de 5,5 cas pour 1000000 d'habitants, avec un total de196 cas déclarés. Ces données comprennent un cas de rougeole chez un voyageur international, qui n'est pas pris en compte dans le dénominateur. Tous les cas ont été confirmés en laboratoire $(29,1 \%, n=57)$ ou présentaient un lien épidémiologique avec un cas confirmé en laboratoire $(70,9 \% ; n=139)$.

La majorité des cas $(99,5 \%, n=195)$ ont été signalés durant la période couvrant les semaines épidémiologiques 1 à 20, qui ont pris fin le 10 janvier et le 23 mai 2015, respectivement. Un maximum de $87(44,4 \%)$ cas a été signalé au cours d'une même semaine, lors d'une éclosion au Québec (semaine 9, qui s'est terminée le 7 mars) (figure 1).

\section{Âge, sexe et lieu}

Des renseignements sur l'âge, sexe et la province ou le territoire de déclaration étaient disponibles pour tous les cas signalés en 2015. L'âge des personnes infectées variait de 1 mois à 55 ans, et l'âge médian était établi à 13,9 ans. Les groupes d'âge les plus souvent signalés étaient celui des jeunes âgés de 10 à 14 ans $(28,1 \%, n=55)$, suivi par celui des personnes âgées de 15 à 19 ans $(19,9 \%, n=39)$ et celui des enfants de 5 à 9 ans 
Figure 1 : Répartition des cas déclarés de rougeole par semaine épidémiologique d'apparition de l'éruption cutanée et par province ou territoire, au Canada, en 2015

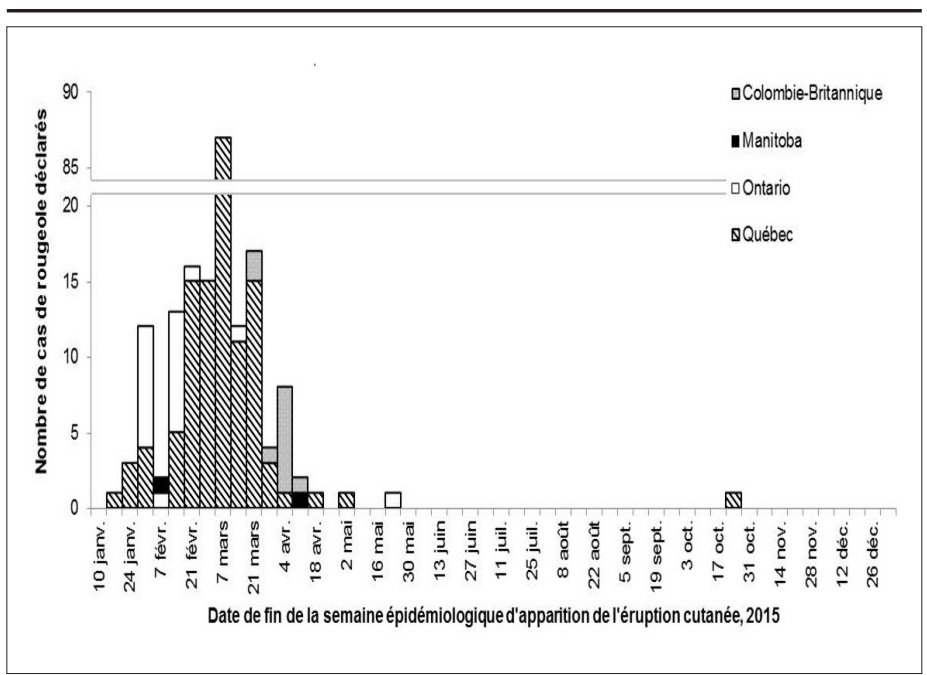

Tableau 1 : Répartition des cas confirmés de rougeole et des taux d'incidence (pour 1000000 d'habitants) par groupe d'âge, sexe et province ou territoire de déclaration ${ }^{1}$, au Canada, en 2015

\begin{tabular}{|c|r|r|r|r|r|r|r|r|}
\hline $\begin{array}{c}\text { Groupe } \\
\text { d'âge }\end{array}$ & H & F & C.-B & MB & ON & OC & CA & $\begin{array}{c}\text { Taux } \\
\text { global } \\
\text { d'incidence }\end{array}$ \\
\hline$<1$ an & 2 & 3 & 0 & 1 & 0 & 4 & 5 & 12,9 \\
\hline 1 à 4 ans & 12 & 6 & 0 & 0 & 4 & 14 & 18 & 11,6 \\
\hline 5 à 9 ans & 22 & 13 & 0 & 0 & 0 & 35 & 35 & 17,9 \\
\hline 10 à 14 ans & 30 & 25 & 1 & 0 & 3 & 51 & 55 & 29,5 \\
\hline 15 à 19 ans & 19 & 20 & 7 & 0 & 1 & 31 & 39 & 18,6 \\
\hline 20 à 24 ans & 6 & 9 & 0 & 0 & 4 & 11 & 14 & 5,7 \\
\hline 25 à 29 ans & 3 & 5 & 0 & 0 & 0 & 8 & 8 & 3,2 \\
\hline 30 à 39 ans & 10 & 4 & 2 & 1 & 5 & 6 & 14 & 2,9 \\
\hline 40 à 59 ans & 4 & 3 & $1+$ & 0 & 3 & 3 & 7 & 0,7 \\
\hline 60 ans et plus & 0 & 0 & 0 & 0 & 0 & 0 & 0 & 0,0 \\
\hline Total & 108 & 88 & 11 & 2 & 20 & 163 & 196 & 5,5 \\
\hline $\begin{array}{c}\text { Taux } \\
\text { d'incidence : }\end{array}$ & 6,1 & 4,9 & 2,3 & 1,5 & 1,5 & 19,7 & 5,5 & \\
\hline Abrivs & & &
\end{tabular}

Abréviations : $\mathrm{H}$, hommes; F, femmes; C.-B, Colombie-Britannique; MB, Manitoba; ON,

Ontario; QC, Québec; CA, Canada

Provinces et territoires où des cas ont été confirmés seulement. Aucun cas de rougeole n'a été signalé en Alberta, au Nouveau-Brunswick, à Terre-Neuve-et-Labrador, dans les Territoires du Nord-Ouest, en Nouvelle-Ecosse, au Nunavut, à l'Íle-du-Prince-Edouard, en Saskatchewan et au Yukon.

${ }^{2}$ Ce dénombrement inclut un cas de rougeole chez un visiteur en Colombie-Britannique qui avait été exposé à bord d'un vol vers le Canada et qui a été incommodé par la maladie au Canada. Ce cas n'apparaît pas dans le bilan provincial de la Colombie-Britannique.
$(17,9 \%, n=35)$. Les taux d'incidence étaient également les plus élevés dans ces groupes, soit $29,5,18,6$ et 17,9 cas pour 1000000 d'habitants, respectivement (tableau 1). Aucun cas n'a été signalé chez les personnes âgées de 60 ans et plus. Environ la moitié des cas signalés $(55,1 \%, n=108)$ étaient des hommes. Quatre provinces canadiennes ont déclaré des cas de rougeole en 2015 : la Colombie-Britannique, le Manitoba, l'Ontario et le Québec. L'incidence était la plus élevée au Québec, suivi par la Colombie-Britannique, le Manitoba et I'Ontario (19,7, 2,3, 1,5 et 1,5 cas pour 1000000 d'habitants, respectivement).

\section{Immunisation}

En 2015 , la vaste majorité des cas $(87,8 \%, n=172)$ n'étaient pas à jour, compte tenu de leur âge, en ce qui concerne le vaccin contenant le virus de la rougeole au moment de l'infection (tableau 2). De même, la majorité des cas $(86,7 \%, \mathrm{n}=170)$ n'avaient jamais reçu de dose du vaccin contenant le virus de la rougeole. Neuf cas de rougeole (cinq nourrissons âgés de moins d'un an et quatre adultes nés avant 1970) n'étaient pas admissibles, en raison de leur âge, à recevoir le vaccin contenant le virus de la rougeole selon les recommandations actuelles sur l'immunisation systématique du Comité consultatif national de I'immunisation (CCNI). Ces cas ont été classés comme étant à jour, sans égard à leurs antécédents de vaccination. Par conséquent, parmi les 16 cas décrits comme étant à jour, seuls sept cas (ou 3,6 \% de tous les cas signalés) avaient déjà reçu le vaccin contenant la rougeole.

Tableau 2 : État vaccinal des cas confirmés de rougeole, par groupe d'âge et exhaustivité de la vaccination ${ }^{1}$, au Canada, en 2015

\begin{tabular}{|c|c|c|c|c|c|c|}
\hline \multirow{2}{*}{$\begin{array}{l}\text { Groupe } \\
\text { d'âge }\end{array}$} & \multicolumn{2}{|c|}{ Non vaccinés } & \multicolumn{2}{|c|}{ Vaccinés } & \multicolumn{2}{|c|}{ Inconnu } \\
\hline & $\begin{array}{l}\text { Pas à } \\
\text { jour }\end{array}$ & À jour & $\begin{array}{l}\text { Pas à } \\
\text { jour }\end{array}$ & À jour & $\begin{array}{l}\text { Impossible à } \\
\text { déterminer }\end{array}$ & À jour \\
\hline$<1$ an & 0 & 5 & 0 & 0 & 0 & 0 \\
\hline 1 à 4 ans & 17 & 0 & 0 & 1 & 0 & 0 \\
\hline 5 à 9 ans & 35 & 0 & 0 & 0 & 0 & 0 \\
\hline 10 à 14 ans & 51 & 0 & 4 & 0 & 0 & 0 \\
\hline 15 à 19 ans & 33 & 0 & 1 & 4 & 1 & 0 \\
\hline 20 à 24 ans & 14 & 0 & 0 & 1 & 0 & 0 \\
\hline 25 à 29 ans & 8 & 0 & 0 & 0 & 0 & 0 \\
\hline 30 à 39 ans & 5 & 0 & 3 & 1 & 5 & 0 \\
\hline 40 à 59 ans & 0 & 2 & 1 & 0 & 2 & 2 \\
\hline $\begin{array}{l}60 \text { ans et } \\
\text { plus }\end{array}$ & 0 & 0 & 0 & 0 & 0 & 0 \\
\hline TOTAL & 163 & 7 & 9 & 7 & 8 & 2 \\
\hline \multicolumn{7}{|c|}{$\begin{array}{l}1 \text { Selon la recommandation actuelle du Comité consultatif national de l'immunisation concernant } \\
\text { l'immunisation systématique, la première dose du vaccin contenant le virus de la rougeole devrait être } \\
\text { administrée à l'âge de } 12 \text { à } 15 \text { mois, et la deuxième dose à } 18 \text { mois ou à n'importe quel moment avant } \\
\text { l'éntrée à lécole (16). Les groupes d'âge pour lesquels il n'y a pas de recommandation sont considérés } \\
\text { comme étant à jour, en ce qui concerne l'âage, même dans les cas n'ayant reçu aucune dose du vaccin } \\
\text { contenant le virus de la rougeole. Cela comprend les nourrissons de moins d'un an, qui sont trop jeunes } \\
\text { pour recevoir le vaccin contenant le virus de la rougeole dans le cadre du calendrier de vaccination } \\
\text { systématique. II n'existe également aucune recommandation pour la plupart des adultes nés avant } 1970,\end{array}$} \\
\hline
\end{tabular}


Aucun des cas signalés n'était né avant 1957, le seuil utilisé dans d'autres pays, comme les États-Unis (15). Selon les données, en 2015, un cas avait reçu trois doses du vaccin contenant le virus de la rougeole. Cependant, la troisième dose avait été administrée dans la semaine précédant l'apparition de l'éruption cutanée et avait vraisemblablement été administrée après l'exposition au virus de la rougeole. L'état vaccinal n'a pu être évalué dans $4,1 \%(n=8)$ des cas, en raison de renseignements manquants.

\section{Hospitalisation}

Dans l'ensemble, en 2015, I'hospitalisation a été indiquée pour $10,7 \%(n=21)$ des cas déclarés (tableau 3$)$. Le plus grand nombre d'hospitalisations a été observé chez les personnes âgées de 20 à 24 ans ( $n=5,33,3 \%$ ). En revanche, la proportion la plus élevée d'hospitalisations s'observe chez les nourrissons âgés de moins d'un an, dont $60 \%(n=3)$ des cas ont été hospitalisés. Presque tous les cas hospitalisés $(95,2 \%, n=20)$ n'avaient aucun antécédent déclaré de vaccination, alors que la plupart des hospitalisations $(76,2 \%, \mathrm{n}=16)$ ont été liées à une éclosion au Québec, dans une communauté religieuse ne pratiquant pas la vaccination. Toutefois, il importe de noter qu'on ignore si $2,6 \%(n=5)$ des cas ont été hospitalisés ou non.

Tableau 3 : Données d'hospitalisation des cas confirmés de rougeole, par groupe d'âge, au Canada, en 2015

\begin{tabular}{|c|c|c|c|c|c|c|c|}
\hline \multirow[b]{2}{*}{$\begin{array}{l}\text { Groupe } \\
\text { d'âge }\end{array}$} & \multirow[b]{2}{*}{ Total } & \multicolumn{2}{|c|}{$\begin{array}{c}\text { Non } \\
\text { hospitalisé }\end{array}$} & \multicolumn{2}{|c|}{ Hospitalisé } & \multicolumn{2}{|c|}{ Inconnu } \\
\hline & & $\mathrm{N}$ & $\%$ & $\mathrm{~N}$ & $\%$ & $\mathrm{~N}$ & $\%$ \\
\hline$<1$ an & 5 & 2 & $40 \%$ & 3 & $60,0 \%$ & 0 & $0,0 \%$ \\
\hline 1 à 4 ans & 18 & 18 & $100 \%$ & 0 & $0,0 \%$ & 0 & $0,0 \%$ \\
\hline 5 à 9 ans & 35 & 33 & $94,3 \%$ & 2 & $5,7 \%$ & 0 & $0,0 \%$ \\
\hline 10 à 14 ans & 55 & 53 & $96,4 \%$ & 1 & $1,8 \%$ & 1 & $1,8 \%$ \\
\hline 15 à 19 ans & 39 & 32 & $82,1 \%$ & 3 & $7,7 \%$ & 4 & $10,3 \%$ \\
\hline 20 à 24 ans & 15 & 10 & $66,7 \%$ & 5 & $33,3 \%$ & 0 & $0,0 \%$ \\
\hline 25 à 29 ans & 8 & 6 & $75,0 \%$ & 2 & $25,0 \%$ & 0 & $0,0 \%$ \\
\hline 30 à 39 ans & 14 & 11 & $78,6 \%$ & 3 & $21,4 \%$ & 0 & $0,0 \%$ \\
\hline 40 à 59 ans & 7 & 5 & $71,4 \%$ & 2 & $28,6 \%$ & 0 & $0,0 \%$ \\
\hline 60 ans et plus & 0 & 0 & - & 0 & - & 0 & - \\
\hline Total & 196 & 170 & $86,7 \%$ & 21 & $10,7 \%$ & 5 & $2,6 \%$ \\
\hline
\end{tabular}

\section{Épidémiologie moléculaire}

En 2015, on disposait d'échantillons pour le génotypage de $28,6 \%(n=56)$ des cas déclarés de rougeole. Cependant, les génotypes ont été déterminés pour tous les événements rougeoleux uniques qui comprennent des éclosions $(n=4)$ et des cas sporadiques sans transmission secondaire $(n=6)$.

Les génotypes détectés étaient les suivants : B3 $(n=23), D 4$ $(n=17), H 1(n=11)$ et D8 $(n=5)$ (figure 2$)$.
Figure 2 : Répartition des génotypes des souches de rougeole détectés en $2015(n=56)$ selon la semaine d'apparition de l'éruption cutanée ${ }^{1}$

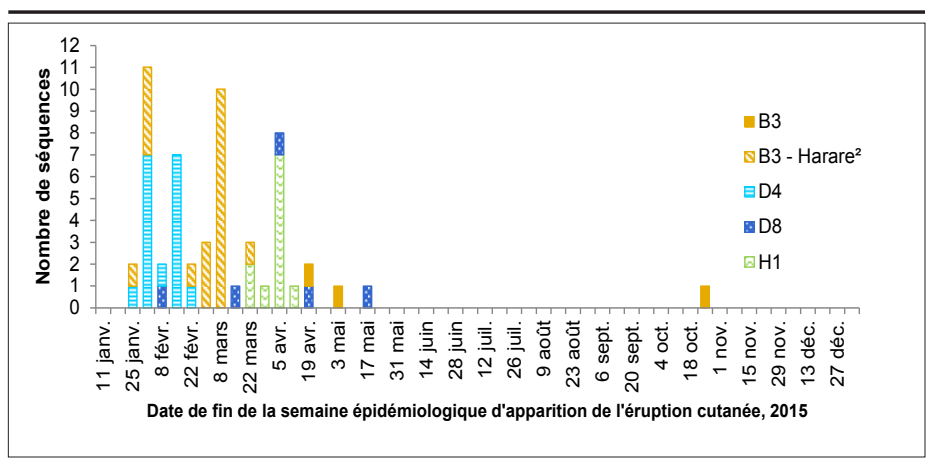

${ }^{1}$ Les semaines épidémiologiques sont attribuées conformément aux lignes directrices de l'Organisation mondiale de la Santé (9), la semaine 1 commençant le premier lundi de chaque année.

${ }^{2}$ Séquences du génotype B3 identiques à la variante MVi/Harare.ZWE/38.09 (numéro d'enregistrement GenBank : JF973033).

Presque toutes les souches du virus identifiées comme étant du génotype $B 3$ étaient identiques à la variante de séquence $\mathrm{MVi} /$ Harare.ZWE/38.09 (numéro d'enregistrement GenBank : JF973033) ( $n=20)$ (figure 3 ). Toutes ces séquences du génotype B3 de variante Harare ont été détectées chez des cas de rougeole associés à l'éclosion au Québec (annexe), qui était liée à une vaste éclosion du génotype $\mathrm{B} 3$ de variante Harare aux États-Unis(17). On a identifié un virus du génotype B3 chez trois autres cas. Alors que ces cas étaient sporadiques et liés à des voyages (en Afrique du Sud, en Éthiopie et en Tunisie), les deux cas avec des antécédents de voyage dans la région de l'Afrique présentaient des séquences $\mathrm{N}$-450 identiques (correspondant à la souche nommée $\mathrm{MVs} /$ Kansas.USA/1.12, numéro d'enregistrement GenBank : JX315576) (figure 3). Toutefois, ces virus de la rougeole se distinguaient par leur séquençage du gène $\mathrm{H}$ (données non indiquées).

Tous les virus du génotype D4 identifiés $(n=17)$ présentaient des séquences $\mathrm{N}-450$ identiques (figure 3) qui n'étaient identiques à aucune souche nommée. Tous ces cas étaient associés à une éclosion d'origine inconnue en Ontario, pour laquelle il a été impossible d'établir un lien épidémiologique entre de nombreux cas (annexe). Afin de mieux caractériser cette éclosion, on a étendu le séquençage, notamment du gène $\mathrm{H}$ et de la région MF-NCR (la région non codante entre la matrice et les gènes chimères). Une description détaillée est à venir.

Des virus du génotype $\mathrm{H} 1$ ont été identifiés dans 11 cas de rougeole, qui avaient tous des antécédents de voyage en Chine, où le génotype $\mathrm{H} 1$ est endémique (11), ou des liens avec un cas ayant voyagé en Chine (annexe). Neuf de ces virus étaient identiques à la souche nommée MVs/Hong Kong.CHN/49.12 (numéro d'enregistrement GenBank : KC417295), alors que les deux autres se distinguaient par un seul nucléotide, mais étaient identiques lorsque comparés l'un à l'autre (figure 3).

Les autres cas de rougeole qui ont été génotypés présentaient tous le génotype D8 $(n=5)$, dont quatre ne concordant avec aucune souche nommée (figure 3). Deux présentaient des séquences $\mathrm{N}-450$ identiques et étaient de la même éclosion associée à des voyages en Inde (annexe). Les trois autres cas de virus du génotype D8 virus étaient des cas sporadiques et présentaient tous des séquences N-450 uniques. Deux cas avaient 
Figure 3 : Arbre phylogénétique des séquences rougeoleuses $\mathrm{N}-450$ détectées au Canada en 2015 $(n=56)$

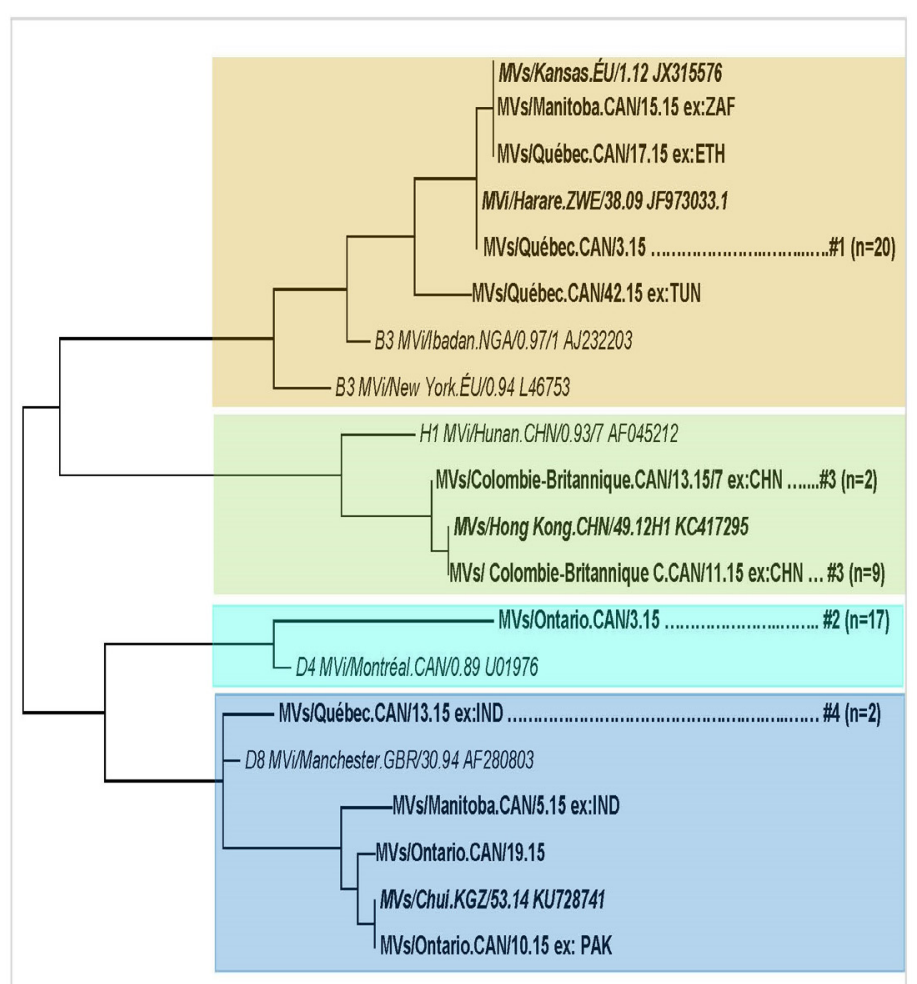

5

REMARQUE : Les séquences de référence pertinentes de l'Organisation mondiale de la Santé (9) sont indiquées en caractères gras italiques. Les souches nommées, assignées dans la base de données des séquences de la rougeole de l'Organisation mondiale de la Santé (MeaNS) [9], sont indiquées en italiques. Les séquences canadiennes sont représentées en caractères ordinaires et sont identifiées par la désignation de l'Organisation mondiale de la Santé qui indique la province et la semaine d'apparition de l'éruption cutanée. Les cas ayant voyagé sont identifiés par « ex: » suivi des trois lettres du code du pays. Les éclosions sont représentées par une seule séquence et sont identifiées par leur numéro d'éclosion (annexe). Le nombre de séquences identiques relevées dans l'éclosion est fourni entre parenthèses. Les séquences restantes, n'ayant aucun numéro d'éclosion, proviennent de cas sporadiques $(n=6)$. L'échelle graphique indique le nombre de différences des nucléotides d'une branche à l'autre.

des antécédents de voyage, I'un en Inde, où le génotype D8 est endémique (11), I'autre au Pakistan, pays voisin du premier, tandis que le troisième cas était de source inconnue. À l'échelle mondiale, le génotype D8 de la rougeole a été le deuxième génotype le plus fréquemment signalé en 2015, selon les soumissions à la base de données Measles Nucleotide Surveillance (MeaNS) de l'OMS (18).

\section{La rougeole canadienne dans le contexte mondial}

En 2015, les cas importés représentaient $4,6 \%(n=9)$ des cas déclarés. Tous les cas importés étaient des adultes (âgés de 16 à 42 ans) qui n'étaient pas complètement immunisés, par rapport à leur âge $(n=7)$, ou des enfants trop jeunes pour être immunisés en vertu du calendrier de vaccination systématique $(n=2)$. Toutefois, étant donné que l'administration du vaccin contenant le virus de la rougeole peut être envisagée dès l'âge de six mois à l'occasion de voyages à l'extérieur de l'Amérique du Nord (16), ces deux enfants représentent également des occasions de vaccination manquées.
Les cas importés ont été exposés à la rougeole lors d'un voyage dans la plupart des régions de l'Organisation mondiale de la Santé : Asie du Sud-Est $(n=2)$, Pacifique occidental $(n=2)$, Méditerranée orientale $(n=2)$, Afrique $(n=2)$ et Amériques $(n=1)$. Aucun des cas importés signalés ne provenait de la région de l'Europe. La Chine et l'Inde étaient chacune à l'origine de deux cas importés déclarés. Un cas importé a été signalé en provenance de chacun des pays suivants : Éthiopie, Pakistan, Afrique du Sud, Tunisie et États-Unis.

En tout, quatre éclosions ont été signalées en 2015, pour un total de 190 cas. La source d'exposition du cas index a été déterminée pour trois des quatre éclosions, provenant de voyages aux États-Unis, en Chine et en Inde. La plus grande éclosion a été occasionnée par un seul cas importé des États-Unis et a compté pour 159 cas au total (annexe). Bien que le nombre de cas signalés pour chaque éclosion variait de 2 à 159 (médiane : 15), la durée des éclosions a été généralement courte, avec une médiane de 3 générations (intervalle de 2 à 6). Les génotypes B3, D4, H1 et D8 ( $n=1$ chaque) ont été identifiés. En 2015, on n'a déterminé aucune source d'exposition pour 14 cas, qui ont tous été signalés par l'Ontario. Treize de ces cas faisaient partie d'une seule éclosion, qui est décrite en détail ailleurs (19). Un seul de ces cas s'est soldé par une propagation secondaire (annexe).

\section{Maintien de l'élimination de la rougeole}

L'Organisation panaméricaine de la Santé a énoncé quatre critères et indicateurs pour la vérification continue de l'élimination de la rougeole (tableau 4). Le Canada a satisfait, pleinement ou partiellement, à trois de ces quatre indicateurs.

\section{Discussion}

Cent quatre-vingt-seize cas confirmés de rougeole ont été déclarés au Canada en 2015, issus de toutes les régions de I'OMS, à l'exception de la région de l'Europe. La majorité de ces cas provenaient d'un seul cas importé associé à une destination touristique populaire aux États-Unis (17). Ce total arrive en troisième position du nombre de cas depuis l'élimination de la rougeole, en 1998, à la suite de celui de $2011(n=725)$ et de celui de $2014(n=418)$. Semblablement à la situation en 2014 , la plupart des cas $(81,1 \%, n=159)$ se trouvaient dans une communauté religieuse ne pratiquant pas la vaccination. Le fardeau était le plus élevé chez les enfants, en particulier chez ceux âgés de 5 à 19 ans, mais aussi chez les enfants âgés de 5 ans ou moins. La plupart des cas hospitalisés n'étaient pas vaccinés. Au moins un cas de chaque événement de rougeole (c.-à-d. quatre éclosions et six cas sporadiques) a été génotypé; quatre génotypes ont été déclarés en 2015, soit B3, D4, H1 et D8. Chaque événement de rougeole était distinct, car tous avaient une souche virale différente. Après la fin de chaque événement, aucune de ces souches virales n'a été observée de nouveau en 2015. La présence de cas dont la source était inconnue laisse croire que tous les cas de rougeole n'ont pas été déclarés; toutefois, le nombre de ceux-ci était relativement faible. Étant donné la durée médiane de l'éclosion de trois générations, toutes les éclosions ont été bien contenues.

Le Canada continue de satisfaire, pleinement ou partiellement, à trois critères sur quatre des indicateurs essentiels de l'Organisation panaméricaine de la Santé pour le maintien 
Tableau 4 : Critères essentiels de l'Organisation panaméricaine de la Santé pour la vérification de l'élimination de la rougeole

\begin{tabular}{|c|c|c|}
\hline Critère & Indicateur & Description \\
\hline $\begin{array}{l}\text { Vérifier } \\
\text { l'interruption des } \\
\text { cas endémiques de } \\
\text { rougeole au cours } \\
\text { d'une période d'au } \\
\text { moins trois ans à } \\
\text { compter du dernier } \\
\text { cas endémique } \\
\text { connu, en présence } \\
\text { d'une surveillance } \\
\text { de qualité } \\
\text { supérieure. }\end{array}$ & $\begin{array}{l}\text { Aucun cas de } \\
\text { transmission } \\
\text { endémique. }\end{array}$ & Critère satisfait. \\
\hline $\begin{array}{l}\text { Maintenir une } \\
\text { surveillance de } \\
\text { qualité supérieure } \\
\text { suffisamment } \\
\text { sensible pour } \\
\text { déceler les cas } \\
\text { importés et ceux } \\
\text { liés à l'importation. }\end{array}$ & $\begin{array}{l}\text { Plus de deux cas } \\
\text { soupçonnés pour } \\
100000 \text { habitants } \\
\text { ont fait l'objet } \\
\text { d'une enquête } \\
\text { adéquate. }\end{array}$ & $\begin{array}{l}\text { Critère partiellement satisfait } \\
\text { Étant donné que seuls les cas } \\
\text { confirmés de rougeole doivent } \\
\text { obligatoirement être déclarés } \\
\text { au Canada, cet indicateur ne } \\
\text { peut être évalué directement. } \\
\text { Toutefois, grâce aux données } \\
\text { du projet pilote sur la } \\
\text { surveillance de la rougeole et } \\
\text { de la rubéole, on a estimé le } \\
\text { taux national d'enquêtes sur les } \\
\text { maladies évoquant la rougeole, } \\
\text { lequel s'est situé entre } 12 \text { pour } \\
100000 \text { habitants (2006, année } \\
\text { sans éclosion) et } 19 \text { pour } \\
100000 \text { habitants (2011, année } \\
\text { touchée par une éclosion) (23). }\end{array}$ \\
\hline $\begin{array}{l}\text { Vérifier l'absence } \\
\text { de souches de } \\
\text { virus endémiques } \\
\text { de la rougeole } \\
\text { en assurant une } \\
\text { surveillance } \\
\text { virologique. }\end{array}$ & $\begin{array}{l}\text { Génotypage de la } \\
\text { rougeole effectué } \\
\text { lors de } 80 \% \text { des } \\
\text { éclosions. }\end{array}$ & $\begin{array}{l}\text { Critère satisfait. } \\
\text { Les renseignements sur le } \\
\text { génotype étaient accessibles } \\
\text { pour } 100 \% \text { des éclosions } \\
\text { signalées en } 2015 .\end{array}$ \\
\hline $\begin{array}{l}\text { Veiller à ce que } \\
\text { l'immunisation } \\
\text { au sein de la } \\
\text { population soit } \\
\text { adéquate. }\end{array}$ & $\begin{array}{l}\text { Quatre-vingt- } \\
\text { quinze pour cent } \\
\text { des cohortes de la } \\
\text { population âgée } \\
\text { de } 1 \text { à } 40 \text { ans ont } \\
\text { reçu un vaccin } \\
\text { contenant le virus } \\
\text { de la rougeole. }\end{array}$ & $\begin{array}{l}\text { Critère non satisfait. } \\
\text { Étant donné qu'il n'existe } \\
\text { actuellement aucun registre } \\
\text { national de vaccination au } \\
\text { Canada, ce critère ne peut pas } \\
\text { être évalué directement. } \\
\text { Toutefois, l'Enquête sur la } \\
\text { couverture vaccinale nationale } \\
\text { des enfants de } 2013 \text { a estimé } \\
\text { à } 89,6 \% \text { le taux de couverture } \\
\text { vaccinale pour la première dose } \\
\text { du vaccin contenant le virus de } \\
\text { la rougeole chez les enfants } \\
\text { âgés de } 2 \text { ans, et à } 85,5 \% \text {, } \\
\text { celui pour la deuxième dose } \\
\text { du vaccin chez les enfants âgés } \\
\text { de } 7 \text { ans (24). Cette estimation } \\
\text { reflète un changement dans la } \\
\text { méthodologie, plutôt qu'une } \\
\text { diminution de la couverture, } \\
\text { par rapport aux années } \\
\text { précédentes (p. ex. } 95,2 \% \text { et } \\
94,9 \% \text { en } 2011 \text { [25]). } \\
\text { II faut noter qu'il s'agit ici } \\
\text { de valeurs moyennes; la } \\
\text { couverture est hétérogène } \\
\text { et peut varier d'une région à } \\
\text { l'autre. }\end{array}$ \\
\hline
\end{tabular}

de l'élimination de la rougeole. Un critère antérieurement satisfait ne l'a pas été en 2015; il s'agit de la couverture par le vaccin contenant le virus de la rougeole. Cette situation reflète probablement un changement dans la méthodologie d'estimation de la couverture, et non une diminution de la couverture réelle. Fait notable, le budget fédéral 2016 prévoit 25 millions de dollars sur cinq ans dans de nouveaux investissements, en soutien à l'amélioration de la couverture vaccinale au Canada (26).

À l'échelle mondiale, l'élimination et l'éradication de la rougeole continuent d'être une priorité en matière de santé publique, toutes les régions de l'Organisation mondiale de la Santé s'efforçant d'atteindre les objectifs d'élimination. Lors de l'Assemblée mondiale de la Santé en 2010, trois cibles pour l'éradication de la rougeole ont également été approuvées en vue d'accroître la couverture par le vaccin contenant le virus de la rougeole et de réduire des taux de morbidité et de mortalité dans le monde au plus tard en 2015 (27). Quoi qu'il en soit, comme les objectifs mondiaux et ceux relatifs à l'élimination n'ont pas été atteints en 2015 (28), il y a encore place à amélioration.

\section{Limites}

Ces données comportent un certain nombre de limites qui méritent un examen. Les indicateurs d'un système de surveillance bien rodé établis par I'Organisation panaméricaine de la Santé sont fondés sur l'investigation des cas évoquant la rougeole (c.-à-d. les cas présumés), alors que seuls les cas confirmés sont à déclaration obligatoire au Canada. À ce titre, ces données ne peuvent qu'indirectement répondre aux critères de l'Organisation panaméricaine de la Santé. En outre, les renseignements sur la mortalité et des renseignements détaillés sur la morbidité (p. ex. durée des hospitalisations, séquelles) ne sont pas actuellement saisis dans le SCSRR ou le projet pilote de surveillance de la rougeole et de la rubéole, ce qui limite la capacité de décrire complètement le fardeau de la rougeole au Canada. Enfin, comme l'état vaccinal est une variable dérivée qui est influencée par les différences des calendriers de l'ensemble des provinces et territoires, il peut être discriminatoire d'une personne à l'autre, en fonction d'un facteur qui ne décrit pas entièrement le risque d'être infectés par le virus de la rougeole.

\section{Conclusion}

Tant au Canada qu'à l'étranger, le maintien d'une couverture élevée par le vaccin contenant le virus de la rougeole demeure un important effort de santé publique, ainsi qu'un élément essentiel d'une stratégie en vue d'atteindre et de maintenir l'élimination de la rougeole. Bien que les cas importés et les zones à faible couverture vaccinale continuent de menacer le statut d'élimination du Canada, les données de surveillance fournissent des preuves solides que l'élimination de la rougeole a été maintenue.

\section{Remerciements}

Les auteurs souhaitent remercier leurs partenaires provinciaux et territoriaux de surveillance et d'analyse en laboratoire pour leur collaboration et leurs efforts continus à fournir et à valider les données saisies dans le SCSRR et le projet pilote sur la surveillance de la rougeole et de la rubéole, à transmettre des 
spécimens pour la surveillance moléculaire (génotypage), ainsi que pour leur revue du contenu de ce rapport.

Les auteurs sont également reconnaissants envers le Dr Alberto Severini, Jennifer Beirnes et Lisa Podhorodecki et le personnel des Services principaux de la génomique du Laboratoire national de microbiologie pour leur contribution dans la production des données moléculaires.

\section{Conflit d'intérêts}

II n'y a aucun conflit d'intérêts à déclarer.

\section{Financement}

Ce travail a été appuyé par l'Agence de la santé publique du Canada.

\section{Références}

1. World Health Organization (WHO). Measles fact sheet N286. Geneva: WHO; November 2015 [updated 2016 Mar]. http:// www.who.int/mediacentre/factsheets/fs286/en/.
2. World Health Organization (WHO). Monitoring progress towards measles elimination. Wkly Epidemiol Rec 2010;85(49):490-494.

3. National Advisory Committee on Immunization (NACl). Statement on elimination of indigenous measles in Canada. Can Dis Wkly Rep 1980;6:33-4.

4. Laboratoire de lutte contre la maladie (LLCM). Conférence de concertation sur la rougeole. Relevé des maladies transmissibles au Canada 1993;19:72-9.

5. Pan American Health Organization (PAHO). The XXIV Pan American Sanitary Conference. Expanded program on immunization - Resolution CSP24.R16. (1994).

6. King A, Varughese P, De Serres G, Tipples GA, Waters J, Working Group on Measles Elimination. Measles elimination in Canada. J Infect Dis 2004 May;189-Suppl 1:S236-42.

7. Agence de la santé publique du Canada. Définitions nosologiques des maladies transmissibles faisant l'objet d'une surveillance nationale. Relevé des maladies transmissibles au Canada 2009;35-Suppl 2:71-2.

8. World Health Organization (WHO). Standardization of the nomenclature for describing the genetic characteristics of wild-type measles viruses. Wkly Epidemiol Rec 1998;73:265.

Annexe : Sommaire des éclosions de rougeole au Canada, classées par date d'apparition de la première éruption cutanée, en 2015

\begin{tabular}{|c|c|c|c|c|c|}
\hline No & Province/Territoire & $\mathrm{n}$ & $\begin{array}{c}\text { Jours } \\
\text { (générations) }\end{array}$ & Génotype & Description \\
\hline 1 & Québec & 159 & $\begin{array}{l}72 \\
\text { (6) }\end{array}$ & B3 - Harare ${ }^{1}$ & $\begin{array}{l}\text { Le cas index de cette éclosion a été exposé à la rougeole lors d'un voyage } \\
\text { à un parc thématique populaire en Californie (États-Unis). } \\
\text { L'infection s'est ensuite propagée dans la communauté religieuse ne } \\
\text { pratiquant pas la vaccination dans laquelle le cas index vivait. } \\
\text { Très peu de cas ont été déclarés à l'extérieur de la communauté religieuse. }\end{array}$ \\
\hline 2 & Ontario & 18 & $\begin{array}{l}23 \\
\text { (3) }\end{array}$ & D4 & $\begin{array}{l}\text { Le cas primaire de cette éclosion n'a pas été trouvé. } \\
\text { Treize cas initiaux dans quatre unités de santé ont été recensés. Ces cas } \\
\text { n'avaient aucun lien épidémiologique entre eux ou avec un cas connu. } \\
\text { Toutefois, selon les dates d'apparition de l'éruption cutanée et les résultats } \\
\text { du génotypage, on suppose qu'ils avaient une source d'exposition } \\
\text { commune. } \\
\text { Un seul de ces cas s'est soldé par une propagation secondaire (à cinq } \\
\text { membres du même foyer). }\end{array}$ \\
\hline 3 & Colombie-Britannique & 11 & $\begin{array}{l}19 \\
\text { (2) }\end{array}$ & $\mathrm{H} 1$ & $\begin{array}{l}\text { Deux cas de rougeole ont été signalés chez des Canadiens qui ont été } \\
\text { exposés au cours d'un voyage en Chine. Ces cas étaient contagieux } \\
\text { pendant le vol de retour au Canada. } \\
\text { Un cas de rougeole exposé durant le vol était un visiteur de la Colombie- } \\
\text { Britannique et n'est pas inclus dans le bilan provincial de cette province. } \\
\text { L'infection s'est ensuite propagée parmi d'autres passagers de ce vol ou } \\
\text { des personnes qui avaient un lien épidémiologique avec le vol. }\end{array}$ \\
\hline 4 & Québec & 2 & $\begin{array}{l}14 \\
\text { (2) }\end{array}$ & D8 & $\begin{array}{l}\text { Le cas index avait des antécédents de voyage en Inde. } \\
\text { Un cas secondaire a été signalé; il avait été exposé au virus de la rougeole } \\
\text { dans un milieu de soins de santé. }\end{array}$ \\
\hline
\end{tabular}


9. Mulders M, Rota P, Brown K, Goodson J. Genetic diversity of wild-type measles viruses and the global measles nucleotide surveillance database (MeaNS). Wkly Epidemiol Rec 2015;90(30):373.

10. Tamura K, Peterson D, Peterson N, Stecher G, Nei M, Kumar S. Molecular evolutionary genetics analysis using maximum likelihood, evolutionary distance, and maximum parsimony methods. Mol Biol Evol 2011;28:2731.

11. Rota PA, Brown K, Mankertz A, et al. Global distribution of measles genotypes and measles molecular epidemiology. J Infect Dis 2011;204-Suppl 1:8514.

12. SAS. Enterprise Guide 5.1. 2013;5.1.

13. Pan-American Health Organization (PAHO). Plan of action. Documentation and verification of measles, rubella and congenital rubella syndrome elimination in the region of the Americas. Technical Document. 2010.

14. Gouvernement du Canada. Programmes de vaccination systématique (et de rattrapage) des provinces et des territoires pour les nourrissons et les enfants au Canada. Ottawa: ASPC; 2016 [Date de modification : le 2 mars 2016]. http://healthycanadians.gc.ca/healthy-living-vie-saine/ immunization-immunisation/schedule-calendrier/infantschildren-vaccination-enfants-nourrissons-fra.php.

15. McLean HQ, Fiebelkorn AP, Temte JL, Wallace GS. Prevention of measles, rubella, congenital rubella syndrome, and mumps, 2013: Summary recommendations of the Advisory Committee on Immunization Practices (ACIP). MMWR 2013;62(RR04):1-34.

16. Comité consultatif national de l'immunisation (CCNI). Guide canadien d'immunisation. Partie 4 : Vaccins actifs vaccin contre la rougeole. Ottawa: ASPC; 2015 [Date de modification le 21 avril 2015]. http://www.phac-aspc.gc.ca/ publicat/cig-gci/p04-meas-roug-fra.php.

17. Zipprich J, Winter K, Hacker J, Xia D, Watt J, Harriman K. Measles outbreak: California, December 2014-February 2015. MMWR 2015;64(06):153-4.

18. World Health Organization (WHO). Measles surveillance data. Geneva; WHO; 2016 [updated 2016 May 18]. http:// www.who.int/immunization/monitoring_surveillance/burden/ $\mathrm{vpd} /$ surveillance_type/active/measles_monthlydata/en/ index1.html.

19. Gournis E, Shane A, Shane E, Arthur A, Berger L. Étude des lacunes dans la surveillance d'une petite éclosion de rougeole à Toronto, Canada. Relevé des maladies transmissibles au Canada 2016;42:155-7.

20. Agence de la santé publique du Canada. Lignes directrices pour la prévention et la contrôle des éclosions de rougeole au Canada. Ottawa: ASPC; 2011.

21. Shane A, Hiebert J, Sherrard L, Deehan H. La surveillance de la rougeole au Canada : Tendances 2013. Relevé des maladies transmissibles au Canada 2014;40(12):244-59.

22. Sherrard L, Hiebert J, Squires S. La surveillance de la rougeole au Canada : Tendances 2014. Relevé des maladies transmissibles au Canada 2015;41(7):181-93.

23. T. EisBrenner. The MARS Pilot Project: Implementing realtime measles and rubella surveillance during elimination phase in Canada [dissertation]. Winnipeg MB: University of Manitoba; 2014.

24. Agence de la santé publique du Canada. Couverture vaccinale des enfants canadiens : points saillants de I'Énquête nationale sur la couverture vaccinale des enfants de 2013 (ENCVE) [Date de modification : le 21 juillet 2015]. Ottawa: PHAC; 2015. http://healthycanadians.gc.ca/ publications/healthy-living-vie-saine/immunization-coveragechildren-2013-couverture-vaccinale-enfants/index-fra.php.

25. Public Health Agency of Canada. Vaccine coverage in Canadian children: Results from the 2011 Childhood National Immunization Coverage Survey. Ottawa: PHAC; 2015.

26. Government of Canada. Budget 2016: Growing the middle class. Chapter 5 - An inclusive and fair Canada. Ottawa: Queen's Printer; 2016. p. 181.

27. Sixty-Third World Health Assembly May 2010, A63/18, Geneva, Switzerland: World Health Organization; 25 March 2010.

28. OMS Groupe stratégique consultatif d'experts (SAGE) sur la vaccination. 2015 Rapport d'évaluation du plan d'action mondial pour les vaccins [mise à jour 2016]. http://www.who. int/immunization/global_vaccine_action_plan/fr/. 